\title{
Phase transitions in Nowak-Sznajd opinion dynamics
}

\author{
Maciej Wołoszyn, Dietrich Stauffer* and Krzysztof Kułakowski \\ Faculty of Physics and Applied Computer Science \\ AGH University of Science and Technology \\ al. Mickiewicza 30, PL-30059 Kraków, Euroland
}

August 3, 2021

* Visiting from Institute for Theoretical Physics, Cologne University, D-50923 Köln, Euroland

e-mail: woloszyn@agh.edu.pl,stauffer@thp.uni-koeln.de, kulakowski@novell.ftj.agh.edu.pl

Abstract: The Nowak modification of the Sznajd opinion dynamics model on the square lattice assumes that with probabilities $\beta$ and $\gamma$ the opinions flip due to mass-media advertising from down to up, and vice versa. Besides, with probability $\alpha$ the Sznajd rule applies that a neighbour pair agreeing in its two opinions convinces all its six neighbours of that opinion. Our Monte Carlo simulations and mean-field theory find sharp phase transitions in the parameter space.

\section{$1 \quad$ Introduction and Model}

A true capital of a country has to coordinate the various regions of that country. In this sense, we apply here the suggestion of Nowak (Warszawa) to modify the Sznajd (Wrocław) model of opinion dynamics.

In the Sznajd opinion dynamics model [1, 2] on the square lattice each site ("agent") can have one of two possible opinions: up (1) or down (0). Two neighbouring sites having the same opinion convince their six neighbours of that opinion. 

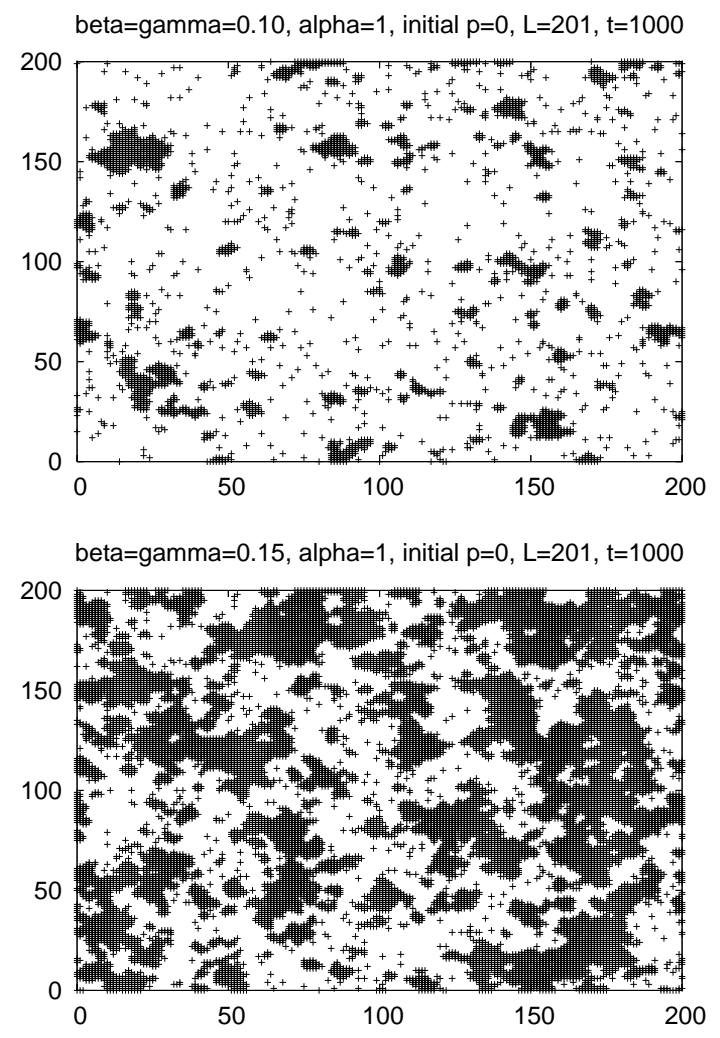

Figure 1: Distribution of up opinions when initially one percent of the agents had opinion up at randomly selected sites. The upper part had a lower flipping probability $\beta=\gamma=0.10$ than the lower part (0.15), and in the lower part the two opinions occur about equally often.

This model simulates the psychological effect that two people in agreement convince better than one person or than two disagreeing people 3. A sharp phase transition results for large lattices if initially a fraction $p$ of the opinions is up randomly: For $p>1 / 2$ at the end everybody has opinion up, and for $p<1 / 2$ everybody ends up with opinion down.

A. Nowak at the GIACS summer school "Applications of Complex Systems to Social Sciences" in September 2006 suggested to generalise this Sznajd model by flipping each up opinion down with probability $\gamma$ and each down opinion up with probability $\beta$, taking into account global effects like advertising through mass media [4. The traditional pair-convincing is applied to each of the six neighbours 

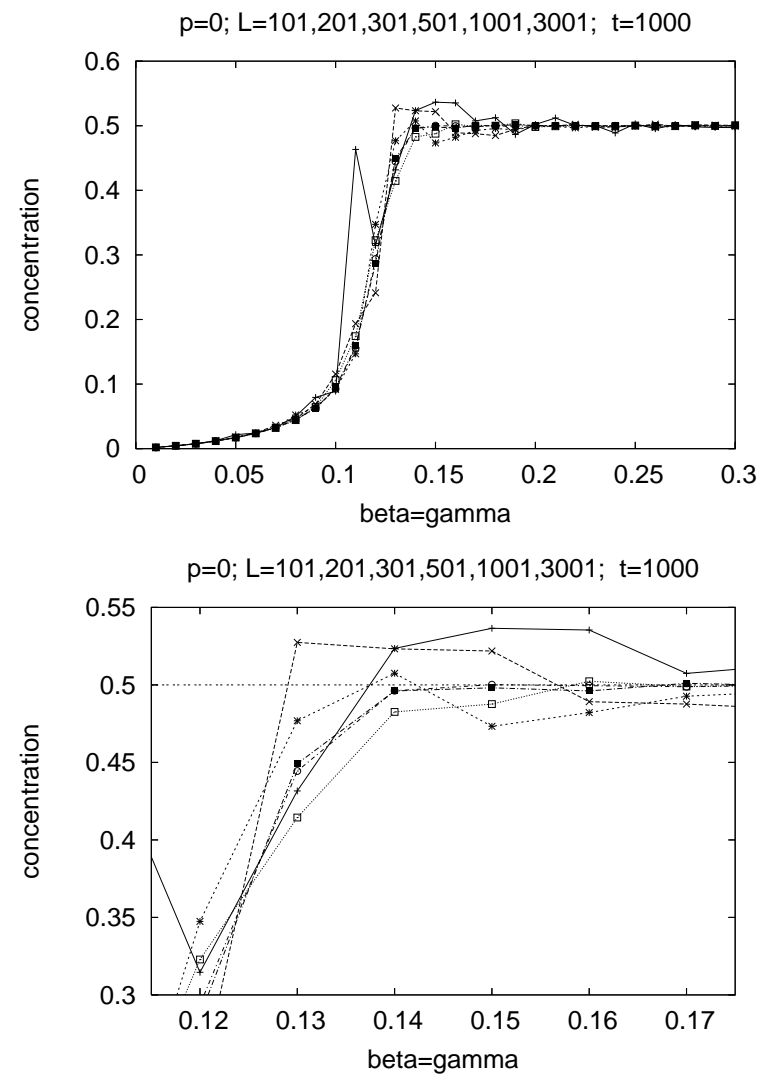

Figure 2: Phase transition when starting from $p=0.01$; one sample was simulated for each point, with $\alpha=1, t=1000, L=101(+), 201$ (x), 301 $(*), 501$ (empty squares), 1001 (full squares) and 3001 (circles).

independently with probability $\alpha$. Obviously, for positive $\beta$ and $\gamma$ no complete consensus is possible anymore, but we can still search for phase transitions where the fraction of up opinions jumps as a function of some continuously varying parameter $(\alpha, \beta, \gamma, p)$.

With $\beta \neq \gamma$ we destroy up-down symmetry; this symmetry can also be destroyed by assuming that only up pairs convince down opinions to flip up: Directed convincing as opposed to the usual undirected convincing.

The next section brings a mean field approximation, and section 3 our simulation results, followed by a concluding section 4 . 


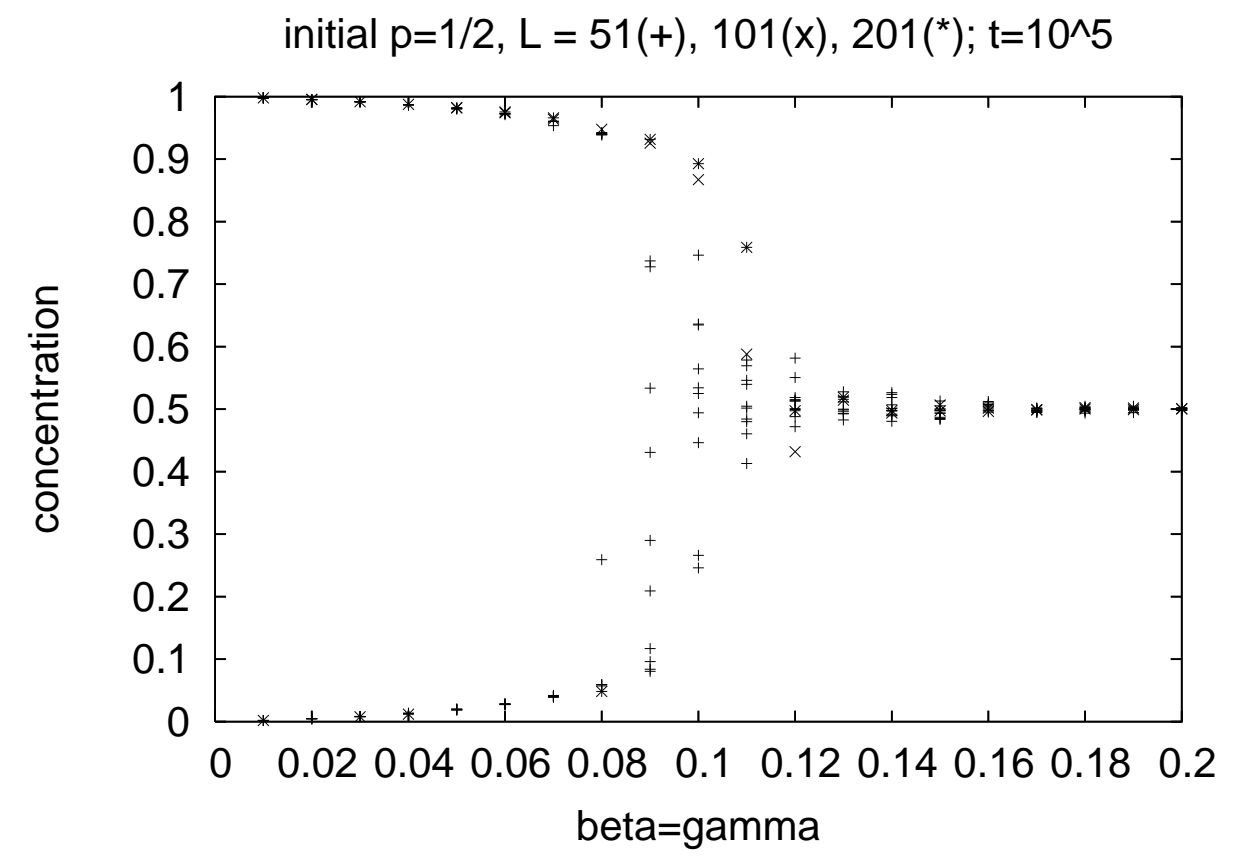

Figure 3: As Fig.2 but starting from $p=0.5$; ten samples each for $L=51$ and one for $L=101$ and $201 ; t=10^{5}$. In some cases the larger lattices seem not yet have found their equilibrium.

\section{Mean-Field Approximation}

In the symmetric case when $\gamma=\beta$, the appropriate Master equation [5] for the probability $p$ of having up opinion, $S=1$, is

$$
\frac{d p}{d t}=-\beta p+\beta(1-p)+\alpha p^{2}(1-p)-\alpha p(1-p)^{2}
$$

In this equation, the first term on the r.h.s. is responsible for spontaneous switching from $S=0$ to $S=1$, the second term - for the opposite, the third - for the switching from 0 to 1 when two neighbours are 1, and the fourth term - for the switching from 1 to 0 when two neighsours are 0 . In the mean field approximation, the spatial neighbouring between those neighbours is neglected. Introducing a quasimagnetisation $m \equiv 2 p-1$ we get

$$
\frac{1}{2} \frac{d m}{d t}=-\beta m+\frac{\alpha}{4} m\left(1-m^{2}\right)
$$




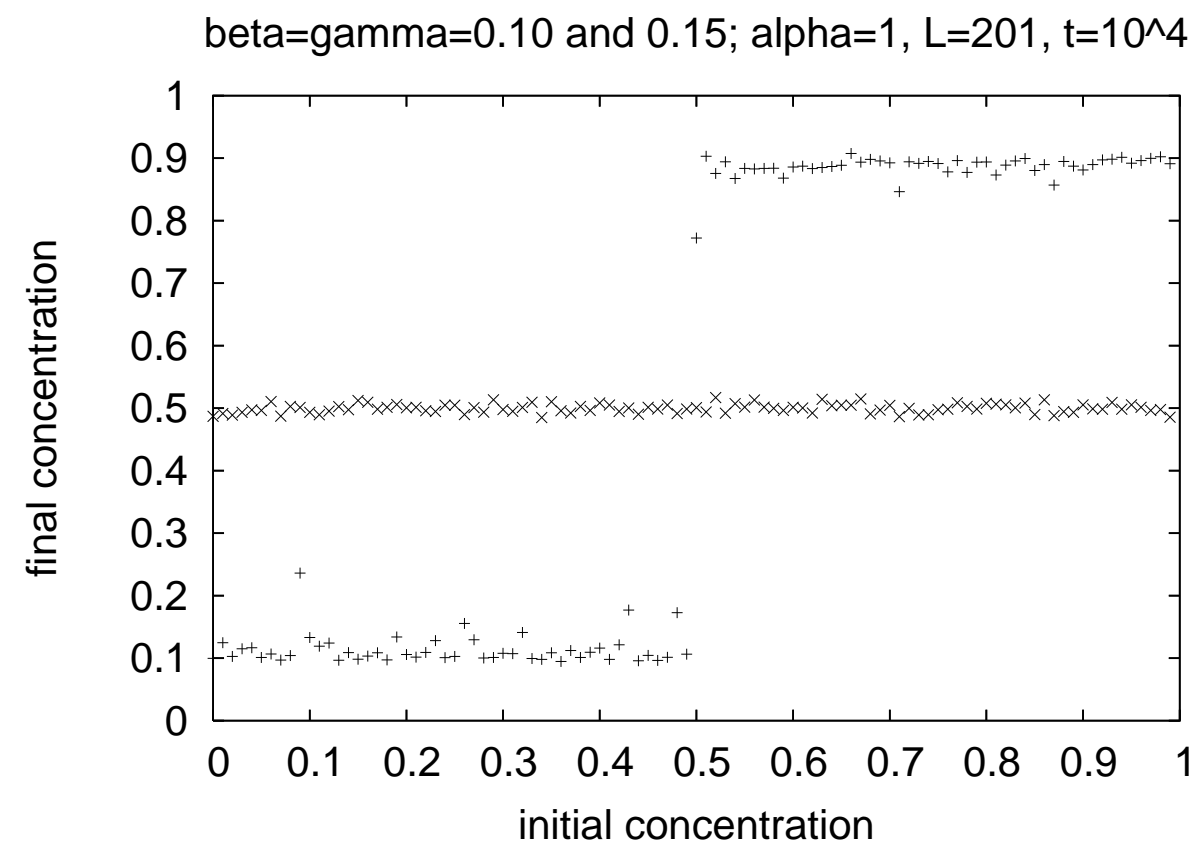

Figure 4: Possible jump as a function of initial concentration if $\beta=\gamma$ is fixed at $0.10(+$, jump) and 0.15 (x, no jump).

For the fixed points $m^{*}$ where $d m / d t=0$, the only relevant is the parameter $x \equiv \beta / \alpha$. As the result we get $m^{*}=0$ or $\pm(1-4 x)^{1 / 2}$. The fixed point is stable if the derivative of the r.h.s. of the last equation with respect to $m$ is negative, and it is not stable if it is positive. Here $m^{*}=0$ is stable if $x>1 / 4$, and the remaining two fixed points exist and are stable if $x<1 / 4$. This is an example of the supercritical pitchfork bifurcation [6]. As we see, the result of the mean field theory is that the phase transition exists and is continuous. The transition point is at $\beta_{c}=\alpha / 4$. Above this value, the spontaneous flipping destroys the correlations between neighbours; those correlations enable the Sznajd process and lead to the ordering. For $\beta=\gamma=0$, the result $m^{*}=0$ or \pm 1 agrees with former mean-field approach to the Sznajd model [7].

\section{Simulations}

Figure 1 shows the final configuration in a $201 \times 201$ square lattice after 1000 iterations (sweeps through the lattice. random sequential updating). We use here 


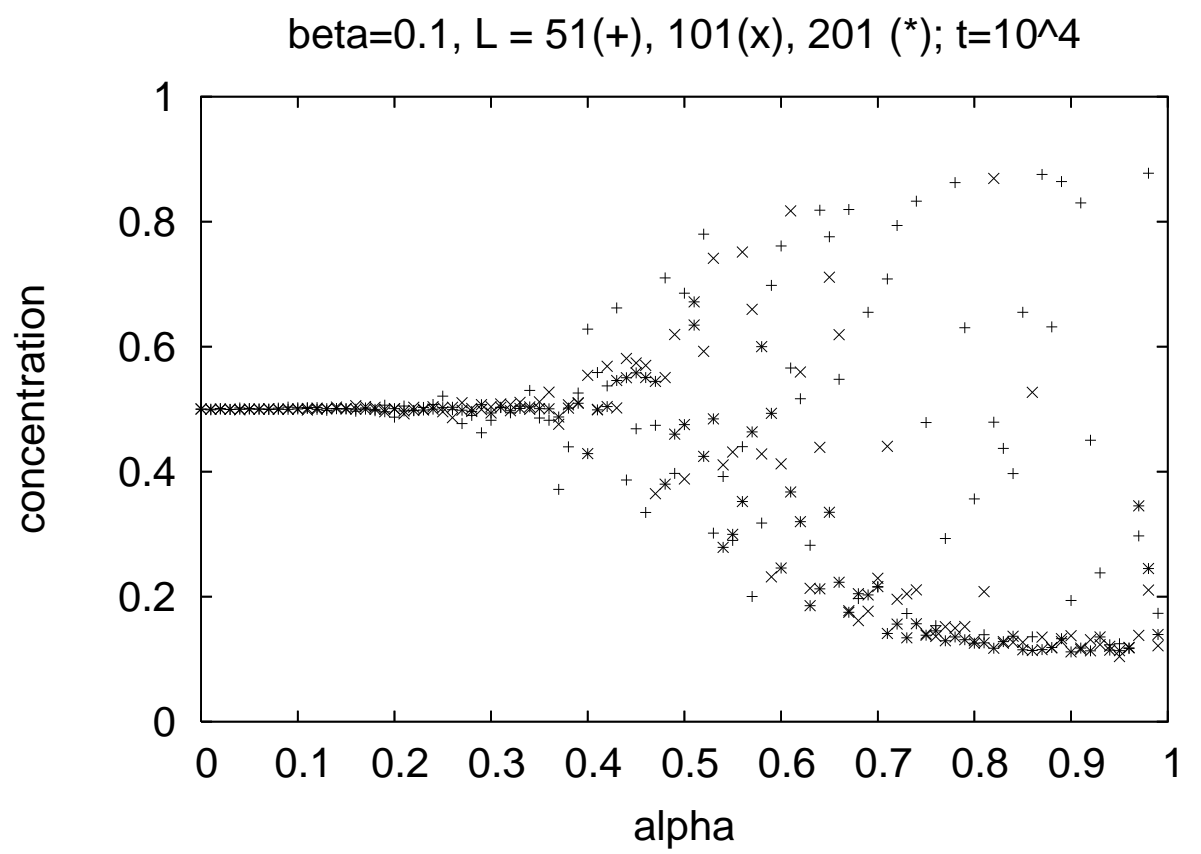

Figure 5: Phase transition as a function of $\alpha$, at fixed $\beta=\gamma=0.1$. Only for $L>100$ a broken symmetry is clearly visible for large $\alpha$.

$p=0.01$ initially and $\alpha=1 ; \beta=\gamma=0.15$ in the upper and 0.20 in the lower part of this figure. Figure 1 shows effects of our helical boundary conditions in horizontal direction, since the clusters ending at the right border are continued on the left border. We see short-range correlations due to the pair-convincing process: The final opinions are not distributed randomly in space even though we started with opinions randomly distributed on the lattice.

Fig. 2 shows more quantitatively the transition between $\beta=\gamma=0.1$ and 0.2 : For low flipping probabilities $\beta, \gamma$ the pair convincing process dominates and most opinions follow the initial majority. For higher flipping probabilities near and above 0.2 the flipping probabilities overwhelm the pair-convincing process and half of the opinions become up, the other half down. We see overlapping curves independent of $L$. The lower part is an expanded plot for the transition region of the upper part. Thus there is a second-order phase transition at $\beta_{c}=\gamma_{c}=0.13 \pm 0.01$ since for a jump (first-order transition) the curves would become steeper and steeper for larger and larger $L$.

The same transition can also be observed starting from $p=1 / 2$, Fig.3, instead 


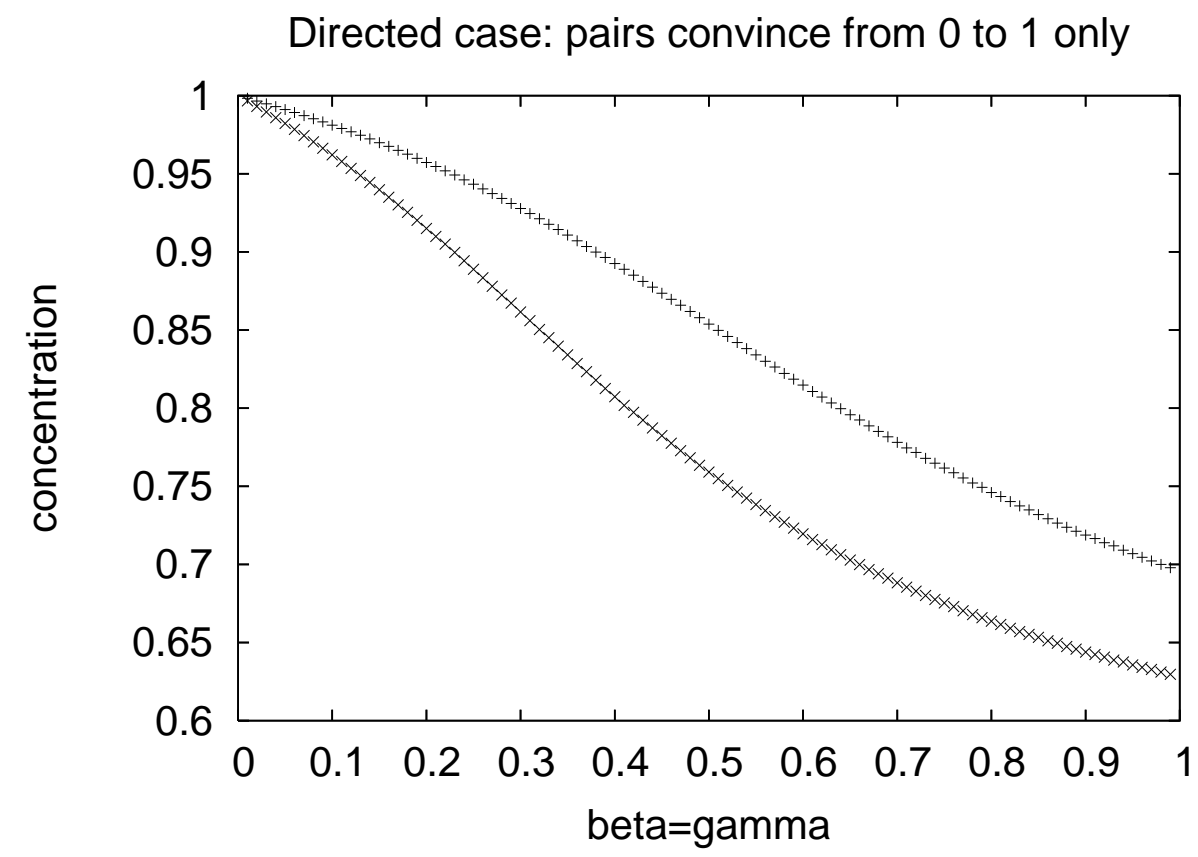

Figure 6: No phase transition when the pair-convincing process works only from 0 to $1 . \alpha=1(+)$ and $1 / 2(\mathrm{x}) ; L=201 ; t=1000$. Results for $L=101$ agree within symbol size with those shown for 201.

of 0.01 as in Fig.2. There, for low flipping probabilities one of the two opinions randomly has to win over the other, which takes more time; thus only smaller $L$ are shown in Fig.3.

In an Ising model, the magnetisation shows a second-order transition, as in Fig.3, if the temperature is varied at zero magnetic field, but a first-order transition (jump) if the magnetic field is varied at constant temperature below $T_{c}$. Somewhat analogously, Fig.4 shows this first-order transition as a function of initial concentration at fixed low $\beta=0.10$ but not at higher $\beta=0.15$.

In all the above figures we had $\alpha=1$; for $\alpha<1$ the pair convincing process not always works, and according to Fig.5 $\alpha>1 / 2$ at $\beta=\gamma=0.1$ is needed to preserve the phase transition (initially, $p=0$ ).

Finally Fig. 6 shows the lack of the phase transition if the symmetric pairconvincing process is replaced by the directed one: Two neighbouring up opinions convince the six neighbours; two neighbouring down opinions convince nobody. We get the same results whether we start from $p=0.01$ or 0.99 , and whether we 
use $L=101$ or 201 . Also if up pairs convince with probability 1 and down pairs convince with probability $1 / 2$, we get the same smooth curve whether we start with $p=0.01$ or 0.09 .

\section{Conclusion}

With a minor addition $(\gamma)$ to the previously simulated Sznajd model with advertising [4] we could see a new second-order phase transition in this opinion dynamics. In contrast to [4 the position of the transition is independent of $L$. On one side the traditional pair-convincing process dominates, on the other side the random opinion flips. This phase transition is predicted by a mean field theory, but the mean-field position of the transition is twice as high as simulated for $\alpha=1$.

We thank A. Nowak for suggesting this work.

\section{References}

[1] K. Sznajd-Weron, J. Sznajd, Int. J. Mod. Phys. C 11 (2000) 1157.

[2] D. Stauffer, S. Moss de Oliveira, P.M.C. de Oliveira and J.S. Sá Martins, Biology, Sociology, Geology by Computational Physicists, Elsevier, Amsterdam 2006

[3] S. Milgram, L. Bickman, and L. Berkowitz, J. Personality Soc. Psych. 13 (1969) 79 .

[4] C. Schulze, C., Int. J. Mod. Phys. C 14 (2003) 95 and C 15 (2004) 569; K. Sznajd-Weron and R. Weron, 2003, Physica A 324 (2003) 437.

[5] C. W. Gardner, Handbook of Stochastic Methods for Physics, Chemistry and the Natural Sciences, Springer-Verlag, Berlin 1983.

[6] P. Glendinning, Stability, Instability and Chaos: an introduction to the theory of nonlinear differential equations, Cambridge UP, Cambridge 1994.

[7] F. Slanina and H. Lavička, Eur. Phys. J. B 35 (2003) 279. 